\title{
INDEX OF SURGEONS AND OTHER MEDICAL MEN
}

A

Abell, Mr., 860

Acton, Mr., 444, 575-576

Adair, Mr., 796, 800, 806, 834

Adair, Mr. Ponsonby R., 792

Adams, Mr., 774, 777, 780, 796, 799, 803, 808, $811,827,886,888,891$

Adams, Mr. W., 738

Adams, Mr. Wm., 693, 757, 768, 797, 835, 881, 891,912

Aikin, Chas., 875

Aikin, Mr., 463

Aikin, Mr. Chas., 109

Alexander, Dr., 579

Alexander, Mr., 481, 724

Alexander, Jnr., Mr., 482

Alleyne, Dr., 290

Amussat, 280

Amussat, M., 794

Ancell, Mr., 463

Anderson, Dr., 449

Anderson, Mr., 446, 538

Angus, Dr., 160

Angus, Mr., 94, 113, 65!

army

medical man named Waring, 771

surgeon, 370

Arnott, Mr., 239, 265, 351, 378, 441, 628-629, 674

Ashburn, Dr., 706

Ashton, Mr., 803, 841, 870, 875, 881

Ashwell, Dr., 664

Attree, Mr., 787

Avery, Mr., 249-250, 277, 287, 289, 292, 299-301, $325,411,431,498,502,516,524$

B

Baber, Dr., 449, 458

Baber, Mr., 517, 887

Bailey, Dr., 271

Baker, Dr., 428

Baldwin, Mr., 27

Barker, Mr., 233

Barlow, Mr. F. W., 396

Barnes, Dr., 173

Barnes, Mr., 379, 845

Bartlett, Mr., 203

Bates, Mr., 517

Beale, Mr., 573, 857

Bell, Dr., 44-45

Bell, Jacob (pharmacist), 43

Bennet, Dr. H., 635

Bennet, Dr. Henry, 97, 870

Bennett, Dr., 128

Billing, Dr., 438, 813, 818, 822

Bird, Dr. Golding, 46, 390

Bird, Mr. James, 842
Blagdon, Mr., 103, 148

Bloxam, Mr., 701

Blundell, Dr., 206, 581

Bowen, Mr., 645

Bowling, Mr., 713

Bowman, Mr., 104, 177, 200, 208, 212, 221, 232, $242,246,257,266,268-269,309,334,340$. $356-357,375,377,383,385,412,429-431,439$, $445,455-456,458,460-462,464,470,477,496$, $500-504,507-508,513-514,523,532-534,540$, $543,552,555-559,561,563-564,566,568-570$, $580-581,583,586-589,594,598,606,608,618$, $627-628,630,632,634,636,638,641,643,648$. $653,655,661,665,673-675,677-678,681,683$, $688,691,694,696-699,703-705,707-708,710$, $712,715-716,725,727-729,731,733-734$, 736-737, 746, 748, 751-754, 756-757, 759-765, $767-768,770-772,777-779,781,785,788-789$, $792-793,795-797,800,802-803,807-810$, $830-832,837,840-842,844-845,847-850$, $858-859,862,864-865,869-871,873,876,878$, $881-882,884,887,889-891,895-901,903-907$, $909,911,913-920$

Braithwaite, Dr., 834

Bramley, Dr., 900

Brigham, Mr., 919

Bright, Dr. James, 507, 621

Brodhurst, Mr., 642, 751, 763, 767, 769, 780, 807-808, 889

Brodie, Sir B., 83, 89, 119, 206, 238, 242, 272, 291, $294-295,358,370,386,390,399,403,436,444$, $450,459-460,537,548,591-592,602,627,685$, $735,757,790,794,839,900$

Brown, Dr., Blakeley, 809

Brown, Mr., 169, 198, 324

Brown, Mr. B., 157-158, 170, 198, 224, 318, 337

Brown, Mr. Baker, 69, 142, 144-145, 181-182, $221,223,332,370$

Brown, Mr. I. B., 368, 398, 530, 775

Bryant, Mr., 417, 542

Bryant, Mr. Walter, 541

Budd, Dr., 587, 589, 599, 756, 793

Bullock, Mr., 399

Buncombe, Mr., 275

Burrows, Dr., 607

Burton, Mr., 861

Busk, Mr., 733

Butler, Mr., 102

Buzzard, Mr., 746, 752

Buzzard, Mr. Thos, 745

C

Cahill, Dr., 303

Cameron, Mr., 839, 865

Cantis, Mr., 360, 602, 670

Canton, Mr., 835, 840, 867 


\section{Index of Surgeons and other Medical Men}

Canton, Mr. Alfred, 584

Canton, Mr. Edwin, 403, 621, 871

Cape, Dr., 461, 563, 609, 649-650, 652, 657, 695, $804-805,869,876$

Ceely, Mr., 757

Chaldecott, Mr., 286

Chalk, Mr., 808

Chambers, Dr. King, 399

Chambers, Dr. K., 327

Chapman, Mr., 732

Charrière, 356

Childs, Mr., 150, 192, 256, 258, 261, 265, 291

Chilver, Mr., 149, 290, 478, 733

Chopart, 24, 57, 172

Chopart, M., 585, 671

Chowne, Dr., 335, 343, 692

Christian, Dr., 446, 623, 713, 775, 837

Clark, Mr. Le Gros, 686

Clark, Sir James, $105,389,448,484,880$

Clarke, Dr. John, 520

Clarke, Mr., 254, 845

Clover, Mr., 668, 685

Coates, Mr., 159

Cochrane, 58

Cock, Mr., 74

Collambell, Mr., 501-502

Cooper, Mr. B., 126, 141, 253, 280, 285, 286-287, $289,339,342,421,695$

Cooper, Mr. Bransby, 83, 122, 197, 204, 224, 233. $238,248,308,337,361,382,384,390,392,443$. 569

Cooper, Mr. White, 306, 339, 362, 380, 384, 388 . $396,404,409-410,429,470,478,483,495,694$. 764,788

Coote, Mr. Holmes, 337

Cornwall, Messrs., 710

Cotton, Dr., 753

Coulson, Mr., 78, 92, 101, 137, 140-141, 147-148, $150,163,170,185-187,191-192,202,206,224$, $244,249,251,254-256,258,268-269,272,275$, $281,290,292,299,302,305,307,312,318$, $320-322,324-325,327,333,337-338,340-342$, $375,718,722,724-726,728,730-732,736-740$, $756-757,770-771,785,795,846,903$

Cowell, Mr., 514

Crampton, Sir Philip, 390

Critchett, Mr., 504, 567, 588, 604, 628, 650, 655, 696, 709, 754

Culverwell, Dr., 6

Cundy, Mr. O., 72

Curling, Mr., 385, 758, 795, 798, 827, 829, 870 , $892,906,911$

Curling, Mr. T. B., 737

Cursham, Dr., 238

Cutler, Mr., 9, 19, 21, 38, 47, 52-53, 60, 68-69, 81, $84-86,89,95,106,113,115,118,228,277,285$, $314,321,323,340,347,350,352,354,370,396$, $403,406,408,413,416-417,425,440-441$, $443-444,446,450,484-485,491,494,514-516$, $573,657,721,766$
$\mathrm{D}$

Dalrymple, Mr., 306, 540

Dampier, Mr., 222, 293

Darling, Dr., 392

Davenport, Mr., 581

Davies, Dr., 453

Davies, Dr. Henry, 723

Davies, Mr., 571

Davis, Dr. J. Hall, 455, 724, 906

Davis, Sir David, 94

Day, Dr., 324

De Meric, Dr., 385

Debout, Dr., 779

Dixon, Dr. Sam, 403

Dixon, Mr., 406, 412

doctor's name omitted by Snow, 447

Dolton, Dr., 727

Ducket, Dr., 913

Duffin, Mr., 31, 58, 122, 279, 303, 577, 635, 677 , $751,758,764$

Dunn, Mr., 91, 388

Dunn, Mr. Rob't, 509-510

\section{E}

Eady, Mr., 595

Edwards, Mr., 460, 577

Elliotson, Dr., 670

Else, Mr., 171

Erichsen, Mr., 11, 366, 826, 863

F

Farish, Mr., 282

Farre, Dr., 451, 454, 458, 678, 687, 721-722, 743 , 799, 856

Farre, Dr. Arthur, 492, 59l

Fell, Dr., 875

Ferguson, Dr., 199, 360, 415, 428, 433, 435, 448, $483,517,614,622,674,874$

Fergusson, Dr. (in Jamaica), 105

Fergusson, Mr., 6, 24, 26, 29, 31-33, 36, 39, 43, 46, $54,57-59,61,68,70,73,76-77,79,83,86$, $90-91,96-97,104-105,108,110-114,116,119$, $121-122,124,126-127,132,134-135,137$, $145-151,153-156,159-160,162,164,166,169$. $171-172,177,181-184,187-192,197,199-200$, 202-204, 206-208, 210-213, 218-225, 229-241, 243-244, 246, 248, 250, 258-265, 267-272, 274, $278-279,283,285-288,290-291,293-294,296$, 298-299, 301-304, 306-313, 315-316, 318-319, $321-331,333-334,336-338,340-346,348-357$, $361,363-380,382,384-389,391-395,397-398$, $400-408,411-413,415,417-418,420-424$, $427-434,436-438,440-448,450,452-453,455$, $457-464,468-472,474-484,486-488,496-500$, $502-505,507-508,510-516,518-520,523-525$, $528,529,533-535,537,539-541,543-548$, $550-552,554-562,564-570,572-576,580-610$, $612-614,617-635,637,639-641,643-649$, 


\section{Index of Surgeons and other Medical Men}

Fergusson, Mr., (cont'd):

653-682, 684-694, 696-713, 715-720, 723-726, $728-733,735-738,740-742,745,747,750,753$, $755,759,762,764,766,768-769,771-772$, $775-776,779,781-785,787-791,794-795$, $797-798,800-802,804-806,809,825-826,828$, 831-846, 848-854, 859-861, 863-873, 875-878, 880-882, 885-892, 895-896, 899-910, 912-915, 917-919

Ferrier, Dr., 2

Filmer, Dr., 601

Foster, Mr., 756

Frampton, Mr., 733

Frankum, Mr., 584

Freeman, Mr., 231

French, Mr., 138, 202, 228-230, 311, 476-477, $554,602,628,743,745,765$

Frere, Dr., 457, 622, 642, 713

Furnival, Mr., 436

G

Garlike, Mr., 206, 209

Gartaway, Mr., 859

Gay, Mr., 165, 260, 280, 369, 382

Genet, Mr., 369, 378, 491

Giles, Mr., 710

Gill, Dr. Battershell, 765

Gillespie, Mr., 652

Giraud, Mr., 438, 568

Girdwood, Dr., 831

Girdwood, Mr., 809, 916

Girdwood, son of Mr., 916

Gloucestershire, surgeon in, 850

Godfrey, Mr., 21, 23

Golding, Dr., 760

Goodday, Mr., 865

Goolden, Dr., 549

Gordon, Dr., 207, 683

Gray, Mr., 514

Gream, Dr., 456-457, 601, 606, 654

Greenhalgh, Mr., 499

Greenhow, Dr. Headlam, 865

Greenwood, Mr., 452

Griffiths, Dr., 355

Guthrie, Mr., 267, 397, 504, 561

Guthrie, Mr. Chas., 665, 692

\section{$\mathrm{H}$}

Hall, Dr. Marshall, 783

Hall, Mr., 913

Hammerton, Mr., 194, 255, 259, 514

Hammick, Sir Stephen, 437

Hancock, Mr., 278, 283, 358, 438, 492, 543, 578 , $584,611,627,672,683,689,694-695,712,719$, $722,753,762,768,832,845,847,872,875,890$, 908,913

Harcourt, Dr., 523, 625

Harding, Mr., 87, 422
Harrison, Mr., 52, 223, 238, 334, 425, 480, 632, 848

Harvey, Dr., 294, 372, 386

Harvey, Mr., 254

Hathaway, Dr., 684

Havers, Mr., 510

Havers, Mr. John, 480

Hawes, Mr., 441

Hawkins, Mr., 19, 25, 27-28, 37-38, 47, 75-78, 89, $95,99,113,118,149,219,263,273,277-278$, $289,345,427,498,572-573,617,661,742,846$, 848

Hawkins, Mr. C., 206

Hawkins, Mr. Caesar, 33, 37, 81, 164, 168-169, $203,217,261,265,270,272,274-275,282,290$, $307,338,344,409,478,480,483,486-487,494$, $510,515,556,606,612,626-627,659,679$, $717-718,723,735,739,760,763,807,830,837$, $875,880,910-911,915$

Hawkins, Mr. Charles, 648

Hawkins, Mr. Chas., 492, 495, 497, 650-653, 685, $703,844,852$

Hayden, Mr., 273

Headland, Mr., 578, 612

Heal, Mr., 515

Heurteloup, M., 273

Hewett, Mr., 12, 28, 33, 35, 37-38, 53, 55, 62, 66, $71,86,94-95,103,219,288-289,315,351,379$. $436,569,576,638,661-662,716,754,848,864$, 869

Hewett, Mr. (of Harrow), 312

Hewett, Mr. P., 705

Hewett, Mr. Prescott, 98, 592, 799

Hewett, Mr. Wm., 297

Hewlet, Mr., 372

Hibbs, Mr., 386

Hicks, Mr., 668-670

Hilton, Mr., 266, 384, 484-485, 604, 666, 739, 760 , 780

Hinxman, Dr., 649

Hird, Mr. Francis, 890

Hodding, Mr., 656

Hodges, Mr., 602

Hodgkin, Dr., 462

Hodgson, Mr., 104, 282, 452, 620, 694, 775

Hogg, Mr., 24l

Holden, Mr., 377

Holland, Sir Henry, 533

Holmes, Mr., 646

Holt, Mr., 732

Holt, Mr. Barnard, 690

Home, Sir Everard, 763

Hood, Mr., 270, 392, 576

Hough, Dr., 224

Houghton, Mr., 438

Hulke, Mr., 748, 800, 806, 838, 854-855

Humble, Dr., 544

Humby, Dr., 571

Hunt, Dr., 633

Hyde, Mr., 371 


\section{Index of Surgeons and other Medical Men}

I

Isaacs, Mr., 167

Isaacs, Jnr., Mr., 167

J

Jakins, Mr., 93

Jefferson, Mr., 626

Jenett, Mr., 528

Johnson, Dr. Ch., 691

Johnson, Dr. George, 917

Johnson, Mr., 6, 9, 12, 19, 25, 27, 31, 35, 41, 43 , $47,53,60,62,67,71-72,75,77-78,82,86,99$, $103,120,164,214,219,392,442,487,573,575$, $716,769,866$

Johnson, Mr. Athol, 37

Johnson, Mr. Chas., 480

Johnson, Mr. H. C., 364, 532, 569, 626, 658-659, 744, 767

Johnson, Mr. H. Chas., 377-378, 392, 766

Johnson, Mr. H. James, 285-286, 338

Johnston, Mr., 238

Jolly, Mr., 155

Jones, Dr., 390

Jones, Mr., 502, 600, 877

Judd, Mr., 185, 275, 314, 448, 452-453, 457, 459, $462,464,470,472$

Julius, Dr., 642,658

Julius, Mr., 164, 247, 349, 568

$\mathrm{K}$

Kane, Mr., 170

Keate, Mr., 9, 27, 33, 50, 65, 80, 89, 94-95, 261, 392

Kent, Mr., 516

Kesteven, Mr., 474

Key, Mr., 27, 74, 112, 119, 192, 535

Key, Mr. Aston, 35, 44-45, 82, 109, 116

Keyser, Mr., 226

Kiernan, Mr., 898

1

Laing, Dr., 376, 410, 504

Lallemand, 89

Lane, Mr., 165, 169, 173, 207, 210, 245, 275, 320 , $345,373,398,445,449,458,463,470,523,538$, $549,879,887$

Lane, Mr. James, 449

Langmore, Mr., 624

Lankester, Dr., 455

Latham, Dr., 490, 794

Laurie, Dr., 776

Laurie, Dr. (homeopath), 381

Lavies, Dr., 567, 628

Lavies, Mr., 243, 679, 684

Lawrence, Mr., 233, 320, 336, 354, 376, 451, $490-491,495,503,688,724,742$
Lawrence, Jnr., Mr. John, 337

Lee, Mr., 232, 237, 240, 307, 313, 354, 387, 409, $415,417,421-425,438,444,492,494,498,599$. $633,650,652,849,854-856,859$

Lee, Mr. H., 317, 668, 742

Lee, Mr. Henry, 98, 215, 220, 267, 299, 366, 383 , $395,399,410,458,480,497,582,587,636,833$, 910

Leese, Dr., 735

Leggatt, Mr., 688

Leonard, Mr., 612

Lever, Dr., 526

Liston, Mr., 123, 207, 480, 546

Little, Dr., 882, 885, 888-890, 919

Lloyd, Dr., 370

Locock, Dr., 381, 448-449, 461, 786

Long, Dr. Henry, 369

Lonsdale, Mr., 765, 768, 774, 777, 780, 796, 799, $804,807-808,811,827,843$

Lucas, Mr., 474

Luke, Mr., 207

Luxton, Mr., 251

M

MacKenzie, Dr., 352, 651

McWilliam, Mr., 236

Malvern, Dr. (homeopath), 119

March, Mr., 368

Markham, Dr., 411

Marsden, Dr., 732

Marshall, Mr., 29, 63, 93-94, 111, 125, 140, $151-152,161,174,193-194,215,226,252,291$, $332,538,562,568,727$

Marshall, Mr. P., 508

Marshall, Mr. Peter, 907

Martin, Mr., 149, 231, 236, 429, 459, 539, 660

Martin, Jnr., Mr., 722

Martin, Mr. Ronald, 691

May, Dr., 739

May, Mr., 112

Mayo, Dr., 99

Mays, Dr., 37

Meadows, Mr., 743, 778

Merriman, Jnr., John, 865

Michelson, Dr., 687

Miller, Dr., 63, 82, 142, 256, 276, 281-282

Moore, Dr., 222

Morgan, Mr., 433, 611

Morgan, Mr. Moses, 241, 719

Morson, Mr. (pharmacist), 330

Morton, Mr., 2, 8-9, 12-13, 36

Mowatt, Mr., 886

Murphy, Dr., 68, 71, 93, 107, 117

Musgrave, Mr., 695, 847

$N$

Nairne, Dr., 133, 480, 650, 668

Newton, Mr., 636, 736 


\section{Index of Surgeons and other Medical Men}

Niell, Mr., 341

Norman, Mr., 214, 216-218

Norman, Mr. B., 451

Noverre, Mr. Arthur, 273

Nunn, Mr., 158, 190

Nurse, Mr., 143

$\mathrm{O}$

O'Connor, Dr., 592

Odling, Mr., 423, 760

\section{$\mathrm{P}$}

Packman, Dr., 312, 366, 671, 684, 826, 850, 884

Page, Dr., 49, 133

Paget, Mr., 377, 489, 504, 508, 520, 522, 539, 574, $597,634,670,685-686,718,720,794,807,864$, 869

Paris, Dr., 481

Parker, Dr., 578

Parkington, Mr., 380

Parkinson, Mr., 67

Parrott, Mr., 105, 770

Partridge, Mr., 24, 31, 39, 54, 56, 61, 79, 90, 110 , $119,135,146,148,157,160,167-168,177,184$, $188,191-192,201,204,206,209,213,219-222$, $229,232,240,246,256-257,260,262,266,274$. $283,288,292,294,304,325,329,343-344,346$, $350,354-357,364,366-367,371,373,375-376$, $378-379,387,389,391,394-395,398,400-402$, $407,451,453,497,572,577,583,589-590,594$, $602,611,617,626,628,639,655,658,660,665$, $673,676,680,690,692,698-699,709,711,715$, $790,792,794,798-799,801,803,806,809,811$, $827,831-832,835-836,839,841,843-844,846$, $849,858,875,897,914,917$

Patterson, Mr., 656

Pearce, Mr., 395

Pearse, Mr., 732

Peirce, Mr., 865

Percy, Dr., 343

Perry, Mr., 422, 592, 674, 690, 704

Pett, Dr., 408

Pettigrew, Dr., 829

Pettigrew, Dr. W. V., 212, 798

Pettigrew, Mr., 176

Pettigrew, Mr. W., 149

Phillips, Mr., 216, 45I

Phillips, Mr. B., 214, 280

Pilcher, Mr., 403

Pirogoff, 891-892, 897

Pitman, Dr., 156

Pollock, Dr., 877

Pollock, Mr., 230, 263, 385, 390, 392, 409, 411, $423,426,440-442,487,518,572-573,575,638$, $657,661,710,721-722,754,848$

Pollock, Mr. G., 344, 539

Pollock, Mr. Geo., 712

Pollock, Mr. George, 837
Pollock, Mr. R. J., 530

Porter, Mr., 916

Potter, Mr., 120, 315, 480, 647

Potter, Mr. (assistant apothecary), 103

Potter, Mr. H., 287

Potts, Mr., 701

Power, Dr., 333

practitioners, un-named, 711,716

Price, Mr., 718, 726, 783, 793, 877, 896

Propert, Mr., 365, 506-507, 586, 627, 768

pupil at St. George's Hospital, 72, 110

Q

Quain, Mr., 42, 123, 187-188, 226, 463-464, 477, $601,645,651,695,719,854,900$

Quin, Dr., 196, 759, 885, 904

$\mathrm{R}$

Radcliffe, Mr., 203

Ramsbotham, Dr., 646-647

Ray, Dr. Septimus, 345

Rees, Dr. Owen, 222, 384

Reid. Dr., 296, 388, 445, 454, 548, 552

Reid, Dr. James, 209

Rendell, Mr. (chiropodist), 339

Reynolds, Mr., 299, 471

Richards, Dr., 112, 689

Richards (medical man), 453

Richardson, Mr., 535

Richardson, Sir John, 243

Ridge, Dr., 339

Rigby, Dr., 548

Roberts, Mr., 287, 71!

Roe, Dr., 341, 371

Roe, Dr. H., 409

Roots, Dr., 101, 107, 369

Rouse, Mr., 101, 114

Routh, Dr., 279

Rowe, Dr., 249, 374

Roy, Mr., 520

Ryan, Dr., 543

$S$

St. George's Hospital pupil, 72, 110

Salmon, Mr., 40I, 453, 456, 458, 462, 478, 493 , $496-497,507,522,527-528,531,536,538,540$, $546,549-551,559,561,569,571-572,577-581$, $594,600,619,623,629,633,635-636,639-640$, $645-646,651,653,655,673,677,683,686$, $695-696,705,727-728,730-731,734,737,743$, $746,757,759-760,769,772-773,782,788,792$, $803,825,830,840,842,845-849,851,859-860$, $862,867,870-871,874-876,882-884,887$, $892-893,908,911,918$

Sampson, Mr., 1, 35, 149, 201, 212, 273

Sankey, Dr., 621

Sawyer, Dr., 664 


\section{Index of Surgeons and other Medical Men}

Scott, Dr., 283-284, 379, 524

Seccombe, Mr., 431

Semple, Dr., 655

Serrell, Mr., 286

Sibley, Mr., 733, 743

Sibson, 101

Sibson, Dr., 143, 158

Simon, Mr., 383

Simpson, Dr., 284

Simpson, Mr., 612

Sison, Mr., 890

Skey, Mr., 602, 828

Smethurst, Dr., 159

Smiles, Dr., 720

Smith, Dr. John Henry, 239

Smith, Dr. Protheroe, 637, 690, 789

Smith, Mr., 142, 321, 323, 342, 382, 405, 475, 595

Smith, Mr. (medical student), 597

Smith, Mr. H., 174, 348, 652

Smith, Mr. Henry, 222, 276, 351, 355, 386, 420,

$561,582,847,854,907$

Solly, Mr., 425, 440, 571. 621, 624, 630, 705, 827

Somerset, Dr., 696

South, Mr., 399, 861

Spitta, Dr., 500, 606, 612

Squibb, Mr., 565

Stanley, Mr., 159-160, 339, 376, 378, 446, 452,

$496,508,526-527,543,602,607,620,676,694$,

$717,735,778,794,898$

Startin, Mr., 508

Stocker, Mr., 50

Stone, Mr., 443

Stracy, Mr., 256

surgeon

army, 370

in Gloucestershire, 850

Sutton, Mr., 399

Sutton, partner of Mr., 399

Sweeting, Mr., 253

Sylvester, Dr., 614

Syme, 96

Syme, Professor, 618, 736

Symes, Dr. E. S., 322

Symonds, Dr., 677

\section{T}

Tamplin, Mr., 376

Tanner, Dr., 828

Tatum, Mr., 19, 32, 37, 66, 84, 89, 91, 109, 118 , $148,210,219-221,315,323,373,379,390,397$, $423,436,572,656-657,721,744,754,852$

Taylor, Mr., 265

Teevan, Mr., 774

Tegart, Mr., 852

Thompson, Dr., 174, 238

Thompson, Dr. Seth, 541

Thompson, Mr. Theophilus, 174, 386

Thomson, Dr., 108
Thorne, Mr., 683

Thudichum, Dr, 625, 702

Todd, Dr., 105, 129, 358, 420, 602, 616, 639, 790 , 800

Toynbee, Mr., 43

Travers, Mr., 163, 180, 184, 602, 613

Travers, Jnr., Mr., 208, 405

Travers, Snr., Mr., 399

Travers, Mr. B., 399, 505, 868

Travers, Jnr., Mr. B., 293, 326, 471, 503, 505, 538

Travers, Mr. Benj., 362

Tuach, Mr., 82, 799

Tuffin, Mr., 225

Turner, Mr., 297, 579

Tweedie, Dr., 451, 724

Tyler-Smith, Dr., 840

$\mathrm{U}$

un-named practitioners, $31,346,376,378,447$, $578,711,716$

see also un-named dentists 569

Ure, Mr., 59, 142, 217, 320, 327, 346

$\mathrm{V}$

Vallance, Mr., 404

Van Oven, Dr., 435, 703, 761, 833, 873

Van Oven, Mr., 241

Verral, Mr., 591

W

Waddell, Mr., 259

Wade, Mr., 111, 142, 149

Wade, Mr. Robert, 366, 651, 664

Wakley, Mr. T., 289, 299

Wall, Mr., 802

Waller, Dr., 206

Walshe, Dr, 556

Walton, Mr., 209, $216,305,312,317,327,331$, $333,338,345-346,368-369,402,534$

Walton, Mr. H., 422, 475, 666, 753

Walton, Mr. Haynes, 189, 206, 621, 725, 734

Ward, Mr. Nathaniel, 720

Wardrop, Mr., 522

Ware, Mr., 678

Waring (army medical man), 771

Warne, Dr., 661

Warry, Mr., 676

Watkins, Messrs, 872

Watson, Dr., 40-41, 122

Webber, Mr., 594, 807

Webber, Mr. Chas., 828

Wells, Mr. Spencer, 214, 477, 733, 735, 770, 779, 804

West, Dr., 886

Wheelwright, Dr., 569

White, Mr., 202

White-Cooper, Mr., 826 
Whitmore, Mr., 523

Wilks, Dr., 581

Williams, Dr., 18, 21

Williams, Dr, C. J. B., 501

Williams, Dr. Caleb, 802

Willis, Dr., 535

Willis, Dr. (of Barrow), 477

Wilson, Dr., 233, 245

Wilson, Mr., 574, 579

Witt, Mr., 850

Wood, Dr., 381, 904

Wood, Mr., 851, 861

Wootton, Mr., 438

Wordsworth, Mr., 758

Wright, Mr., 670

Y

Yorke, Mr., 558

Young, Dr., 105

Young, Mr., 400, 670, 686, 794 